\title{
Taxonomic Note: a Proposal for Reviewing the Interpretation of the CAMP Reaction between Listeria monocytogenes and Rhodococcus equi
}

\author{
J. F. FERNÁNDEZ-GARAYZÁBAL, G. SUÁREZ, M. M. BLANCO, \\ A. GIBELLO, AND L. DOMÍNGUEZ* \\ Departamento Patología Animal I (Sanidad Animal), Facultad de Veterinaria, \\ Universidad Complutense, 28040 Madrid, Spain
}

\begin{abstract}
The discrepancies between the current description of the CAMP test between Listeria monocytogenes and Rhodococcus equi in the latest edition of Bergey's Manual of Determinative Bacteriology (L. monocytogenes is described as CAMP test negative with $R$. equi) and routine findings (positive reactions are usually described in many laboratories) make it advisable to review the current interpretation of the CAMP test to avoid confusion among people working in microbiological laboratories. Overall, $98.4 \%$ of the $L$. monocytogenes strains examined in this study, regardless of their source or the intensity of their hemolytic activity, displayed a synergic hemolytic reaction (CAMP phenomenon) with $R$. equi, indicating that $L$. monocytogenes can generally be considered CAMP positive with $R$. equi. We propose that $L$. monocytogenes, together with Listeria ivanovii, should be considered CAMP test positive with $R$. equi (circular or racket and semicircular or shovel shapes, respectively).
\end{abstract}

Despite the numerous tests of phenotypic and genotypic character that have appeared in the scientific literature in the last years, hemolytic activity, together with the acidification pattern of a few sugars, is considered an essential criterion for the characterization and identification of the different species of Listeria $(13,16)$. As the interpretation of the hemolytic activity of Listeria strains on blood agar plates is sometimes problematic (2), a synergistic hemolytic reaction (CAMP test) with Staphylococcus aureus and Rhodococcus equi was proposed (11). The CAMP test is considered to be a fundamental taxonomic criterion (13), being especially useful for differentiating between very weakly hemolytic strains of Listeria monocytogenes and nonhemolytic strains of Listeria innocua, as both species are very similar biochemically and are frequently isolated simultaneously. In the latest edition of Bergey's Manual of Determinative Bacteriology, L. monocytogenes and Listeria seeligeri are described as CAMP positive with $S$. aureus and CAMP negative with $R$. equi (SA+/RE-). The typical CAMP reaction of Listeria ivanovii is $\mathrm{SA}-/ \mathrm{RE}+$, while the CAMP reaction for both $L$. innocua and Listeria welshimeri is SA-I $\mathrm{RE}-(13)$.

While the interpretation of the CAMP test between $S$. $a u$ reus and $L$. monocytogenes has been widely accepted, the interpretation of the CAMP test between $L$. monocytogenes and $R$. equi is more controversial. In spite of the fact that $L$. ivanovii is the single species of Listeria considered CAMP positive with $R$. equi, we have reported clear synergistic hemolytic reactions (CAMP test positive) with many L. monocytogenes strains of different origin (collection, clinical, environment, food, and feedstuffs), indicating the reproducibility of the CAMP phenomenon between both species $(4,16-19)$. Similar cooperative hemolytic reactions are observed when diffusion CAMP assays are performed with supernatant cultures of these species (3). Table 1 shows the $R$. equi CAMP results with different collec-

* Corresponding author. Mailing address: Dpto. Patología Animal I (Sanidad Animal), Facultad de Veterinaria, Universidad Complutense, Avda. Puerta de Hierro s/n, 28040 Madrid, Spain. Phone: 44 13943721 . Fax: 4413943908. tion strains and field isolates of $L$. monocytogenes. Overall, $98.4 \%$ of the $L$. monocytogenes strains gave a positive CAMP reaction with $R$. equi. Only 6 of 373 strains, identified biochemically as $L$. monocytogenes, were nonhemolytic and were also CAMP test negative with $R$. equi, while we have not found any hemolytic strain of $L$. monocytogenes that gives a negative CAMP reaction with $R$. equi. Positive CAMP reactions between $L$. monocytogenes and $R$. equi have frequently been reported by other authors as well $(7,8,10,12,14,20)$. The CAMP reactions of $L$. monocytogenes and $L$. ivanovii with $R$. equi differ in the shape of the zone of synergic hemolysis. The CAMP test between $L$. ivanovii and $R$. equi produces a semicircular or shovel shape, while the CAMP test between $L$. monocytogenes and $R$. equi displays a circular or racket shape $(16,17)$ (Fig. 1). We have found total reproducibility in performing the CAMP test on Columbia sheep blood agar base (Biomerieux) with $R$. equi STCC (Spanish Type Culture Collection) 4568 after 24 to $48 \mathrm{~h}$ of incubation at $37^{\circ} \mathrm{C}(16)$.

The exosubstances involved in the synergistic hemolytic reaction of $L$. monocytogenes and $L$. ivanovii with $R$. equi have been identified. The CAMP phenomenon between $L$. ivanovii and $R$. equi involves a sphingomyelinase $\mathrm{C}$ and a cholesterol oxidase, respectively (10). Listeriolysin O (LLO) is the cytolytic factor of $L$. monocytogenes involved in the CAMP reaction with $S$. aureus and $R$. equi (10), while the enzymes of $S$. aureus and $R$. equi implicated in the cooperative hemolytic reaction with L. monocytogenes are, respectively, a sphingomyelinase and a cholesterol oxidase $(3,7)$.

Two reasons have usually been argued to reject a positive CAMP reaction between $L$. monocytogenes and $R$. equi. First, this CAMP reaction does not display the typical shovel-like shape observed with $L$. ivanovii, and second, many of the investigators reporting positive CAMP reactions for $L$. monocytogenes used strains of $R$. equi different from the collection strain CIP 5869 indicated by Rocourt et al. (11). Regarding the shape of the CAMP reaction, now that the exosubstances involved in the CAMP reaction between $L$. monocytogenes and $R$. equi have been determined, the fact that the CAMP reaction between both species does not display a typical shovel-like 
TABLE 1. Hemolytic activity and CAMP reaction with $R$ equi of different collection strains and field isolates of $L$. monocytogenes ${ }^{a}$

\begin{tabular}{|c|c|c|c|}
\hline Source & Serovar & $\begin{array}{l}\text { Hemolytic } \\
\text { activity }^{b}\end{array}$ & $\begin{array}{l}\text { CAMP reaction } \\
\text { with } R . e q u i^{b}\end{array}$ \\
\hline \multicolumn{4}{|l|}{ Collection strains ${ }^{c}$} \\
\hline NCTC 8817 & $\mathrm{ND}^{d}$ & + & + \\
\hline NCTC 4885 & ND & ++ & + \\
\hline NCTC 5105 & $3 a$ & +++ & + \\
\hline NCTC 7973 & $1 / 2 \mathrm{a}$ & ++ & + \\
\hline NCTC 5214 & $4 a$ & + & + \\
\hline NCTC 5348 & $1 / 2 \mathrm{c}$ & + & + \\
\hline SLCC 3551 & $4 \mathrm{~b}$ & $+1-$ & + \\
\hline SLCC 2482 & 7 & + & + \\
\hline SLCC 2378 & $4 \mathrm{e}$ & + & + \\
\hline NCTC $10357^{e}$ & $1 / 2 \mathrm{a}$ & - & - \\
\hline SLCC 4324 & $4 \mathrm{~b}$ & + & + \\
\hline SLCC 5132 & $1 / 2 \mathrm{a}$ & + & + \\
\hline Scott A & $4 \mathrm{~b}$ & ++ & + \\
\hline \multicolumn{4}{|l|}{ Clinical isolates } \\
\hline Encephalitis in sheep $(27)^{f}$ & $4 \mathrm{~b}$ & $+1++(26)^{\prime}$ & $+(26)^{f g}$ \\
\hline \multicolumn{4}{|l|}{ Feed/Foods isolates } \\
\hline Silage (30) & $4 \mathrm{~b}$ & $+/++(29)$ & $+(29)^{g}$ \\
\hline Chicken (12) & ND & $+/++(12)$ & $+(12)$ \\
\hline Soft cheese (40) & ND & $+/++(40)$ & $+(40)$ \\
\hline Frozen foods (51) & ND & $+/++(48)$ & $+(48)^{g}$ \\
\hline \multicolumn{4}{|l|}{ Environmental isolates } \\
\hline Slaughterhouse (179) & ND & $+/++(178)$ & $+(178)^{g}$ \\
\hline Fish industry (28) & ND & $+/++(22)$ & $+(22)$ \\
\hline
\end{tabular}

${ }^{a}$ Data obtained from L. monocytogenes strains isolated in our laboratory and references 4,17 , and 19 .

${ }^{b}$ Hemolytic activity and R. equi CAMP reaction were determined in Columbia sheep blood agar. The hemolytic activity in the clinical, feed/food, and environmental isolates is an estimation of the hemolysis average displayed by all the strains included in each group. $+/-$, very weak hemolysis (sometimes it is necessary to remove the colonies to see the zone of hemolysis); +, weak hemolysis; ++ , moderate hemolysis; +++ , strong hemolysis.

' NCTC, National Collection of Type Cultures; SLCC, Special Listeria Culture Collection.

${ }^{d} \mathrm{ND}$, not determined.

${ }^{e}$ Nonhemolytic type strain of $L$. monocytogenes (5). As LLO is involved in the CAMP phenomenon, the nonhemolytic strains of $L$. monocytogenes do not display RE+ CAMP reactions.

$f$ The number of strains that were analyzed, that were hemolytic, and that displayed RE+ CAMP reactions are shown in parentheses (as appropriate).

${ }^{8}$ Six strains of $L$. monocytogenes (identified biochemically) isolated from a clinical case of encephalitis in sheep (1), a slaughterhouse environmental sample (1), silage (1), and frozen foods (3) were nonhemolytic and were also CAMP test negative with $R$. equi.

shape should not be the reason to consider this test negative. Weakly, moderately, and strongly hemolytic strains of $L$. monocytogenes give posivite CAMP reactions with $R$. equi (Table 1). Keeping in mind that LLO is involved in this synergistic hemolytic reaction, the variations in the size of the zone of synergic hemolysis that are observed with different $L$. monocytogenes strains are probably associated with variations in the ability of these strains to secrete LLO (Fig. 1 and 2). Weakly hemolytic strains of $L$. monocytogenes display positive CAMP reactions that are smaller than those produced by stronger hemolytic strains. This fact can be clearly observed in Fig. 1, in which L. monocytogenes NCTC 8817 (weakly hemolytic) displays a CAMP reaction that is smaller than that of $L$. monocytogenes NCTC 7973, while the reaction of the latter strain is smaller than that produced by L. monocytogenes NCTC 5105 (strongly hemolytic) (Fig. 2). Only nonhemolytic strains of $L$. monocytogenes (e.g., strain NCTC 10357 in Fig. 2) were CAMP negative (as expected considering that LLO is implicated in the synergistic hemolytic reaction). The great majority of the L. monocytogenes isolates, regardless of their source, show weak or moderate hemolysis (Table 1) yet display clear RE+ CAMP reactions (Fig. 2). Despite these differences in the size of the CAMP reaction, it seems clear that there is a synergistic hemolytic reaction (CAMP phenomenon) with most of the $L$. monocytogenes strains in their intersection with $R$. equi. From these results it can be stated that $L$. monocytogenes can generally be considered CAMP positive with $R$. equi. In fact, from our experience, the high reproducibility of the CAMP reaction with $R$. equi makes it especially useful as one of the confirmatory tests to differentiate strains of $L$. monocytogenes from $L$. innocua, particularly in cases of weakly hemolytic strains of $L$. monocytogenes (Fig. 2), which display unambiguously positive CAMP reactions with $R$. equi, while $L$. innocua strains are, as expected, RE-

The differences in intensity reported in the CAMP reaction with $L$. monocytogenes depending on the $R$. equi strain used to perform the test $(8,12)$ may be explained by variations in the capability of different strains of $R$. equi to secrete cholesterol oxidase (15). Weak CAMP reactions observed with some strains of $R$. equi, specially with very weakly hemolytic strains of $L$. monocytogenes, can be clearly detected by extending the incubation period (18). In practice, the fact that there are not specific recommendations for using a particular strain of $R$. equi in some of the most common manuals of clinical or food microbiology $(1,6,9,20)$ means that many laboratories, using different strains of $R$. equi, usually find positive CAMP reactions for $L$. monocytogenes $(7,8,10,14,16,17-20)$. It could be stated, therefore, that among Listeria spp., a positive CAMP test with $R$. equi is not exclusively limited to $L$. ivanovii, as it is currently considered to be.

As the CAMP reaction is still a very useful and widely used test for the routine identification of Listeria spp. $(13,16)$, and in order to avoid confusion among people working in clinical and food microbiology laboratories, the current interpretation of the CAMP test between $L$. monocytogenes and $R$. equi should be reviewed. We propose that both $L$. monocytogenes and $L$. ivanovii should be considered CAMP test positive with

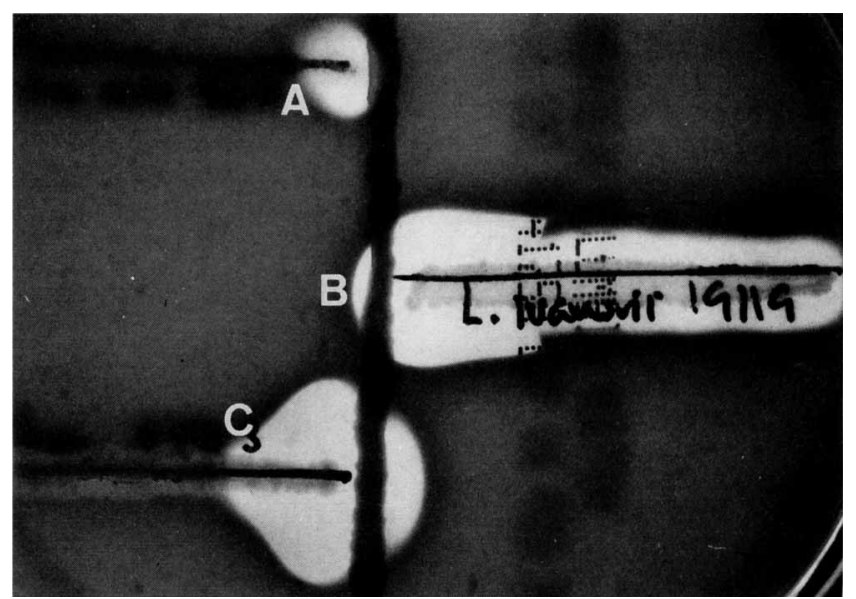

FIG. 1. CAMP reactions with $R$. equi STCC 4568 (vertical streak of growth) and L. monocytogenes NCTC 8817 (A), L. ivanovii ATCC 19119 (B), and L. monocytogenes NCTC 7973 (C) on blood agar plates after $48 \mathrm{~h}$ of incubation at $37^{\circ} \mathrm{C}$. Both $L$. monocytogenes and $L$. ivanovii display synergistic hemolytic reactions (positive CAMP test) with $R$. equi, although with different shapes. $L$. ivanovii displays the typical semicircular or shovel shape, while $L$. monocytogenes produces a circular or racket-shaped CAMP reaction of different size depending of the strain of L. monocytogenes tested. 


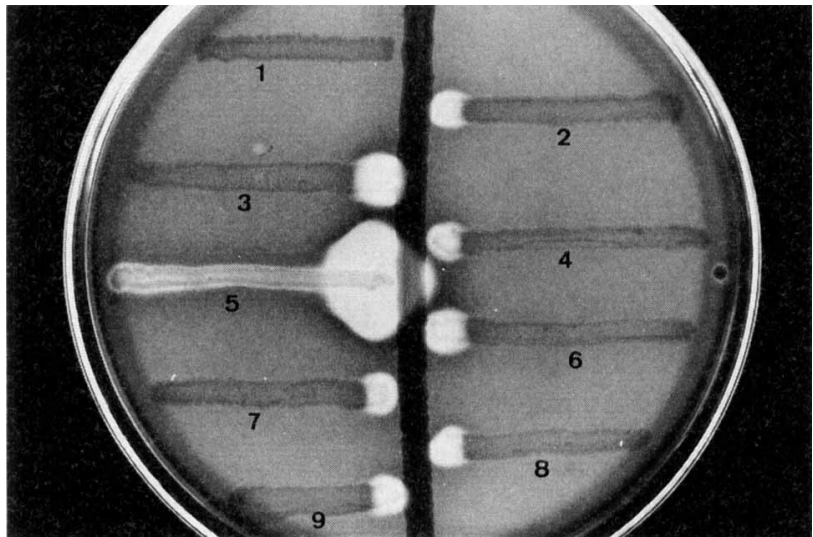

FIG. 2. CAMP reactions with $R$. equi displayed by different collection strains and field isolates of $L$. monocytogenes. The isolates used are as follows: $L$. monocytogenes NCTC 10357 (1), L. monocytogenes SLCC 3551 (2), L. monocytogenes SLCC 2482 (3), L. monocytogenes SLCC 4324 (4), L. monocytogenes NCTC 5105 (5), L. monocytogenes isolated from a case of encephalitis (6), L. monocytogenes isolated from silage (7), L. monocytogenes isolated from frozen minced meat (8), and $L$. monocytogenes isolated from a slaughterhouse environment (9). Most of the field isolates, as well as the collection strains, of $L$. monocytogenes are weakly or moderately hemolytic but nevertheless display clearly positive CAMP reactions with $R$. equi.

R. equi (circular or racket and semicircular or shovel shapes, respectively), while $L$. monocytogenes (as well as $L$. seeligeri) is CAMP positive with $S$. aureus. Thus, L. monocytogenes and L. ivanovii should be described as being $\mathrm{SA}+/ \mathrm{RE}+$ and $\mathrm{SA}-/$ $\mathrm{RE}+$, respectively, for the CAMP test.

\section{REFERENCES}

1. Bille, J., and M. P. Doyle. 1991. Listeria and Erysipelothrix, p. 287-295. In A Balows, W. J. Hausler, Jr., K. L. Herrmann, H. D. Isenberg, and H. J. Shadomy (ed.), Manual of Clinical Microbiology, 5th ed. American Society for Microbiology, Washington, D.C.

2. Domínguez Rodríguez, L., J. A. Vázquez-Boland, J. F. Fernández-Garayzábal, P. Echalecu Tranchant, E. Gómez-Lucia, E. F. Rodíguez Ferri, and G. Suárez Fernández. 1986. Microplate technique to determine hemolytic activity for routine typing of Listeria strains. J. Clin. Microbiol. 24:99-103.

3. Fernández-Garayzábal, J. F., C. Delgado, M. M. Blanco, G. Suárez, and L Dominguez. 1996. Cholesterol oxidase from Rhodococcus equi is likely the major factor involved in the cooperative lytic process (CAMP reaction) with Listeria monocytogenes. Lett. Appl. Microbiol. 22:249-252.

4. Franco Abuin, C. M. 1993. Ph.D. thesis. University of Santiago de Compostela, Lugo.

5. Jones, D., and H. P. R. Seeliger. 1983. Designation of a new type strain for Listeria monocytogenes, request for an opinion. Int. J. Syst. Bacteriol. 53:429.

6. Lovett, J. 1989. Listeria monocytogenes, p. 283-310. In M. P. Doyle (ed.), Foodborne bacterial pathogens. Marcel Dekker, Inc., New York.

7. McKellar, R. C. 1994. Use of the CAMP test for the identification of Listeria monocytogenes. Appl. Environ. Microbiol. 60:4219-4225.

8. Nakazawa, M., and H. Nemoto. 1980. Synergistic hemolysis phenomenon of Listeria monocytogenes and Corynebacterium equi. Jpn. J. Vet. Sci. 42:603607.

9. Quinn, P. J., M. E. Carter, B. Markey, and G. R. Carter. 1994. Listeria species, p. 170-174. In P. J. Quinn, M. E. Carter, B. Markey, and G. R. Carter (ed.), Clinical veterinary microbiology. Wolfe, London.

10. Ripio, M. T., C. Geoffroy, G. Domínguez, J. E. Alouf, and J. A. VázquezBoland. 1995. The sulphydril-activated cytolysin and a sphingomyelinase C are the major membrane-damaging factors involved in cooperative (CAMPlike) haemolysis of Listeria spp. Res. Microbiol. 146:303-313.

11. Rocourt, J., A. Schrettenbrunner, and H. P. R. Seeliger. 1983. Différentiation biochimique des groupes génomiques de Listeria monocytogenes (sensu lato). Ann. Microbiol. (Paris) 134A:65-71.

12. Schuchat, A., B. Swaminathan, and C. V. Broome. 1991. Epidemiology of human listeriosis. Clin. Microbiol. Rev. 4:169-183.

13. Seeliger, H. P. R., and D. Jones. 1986. Genus Listeria Pirie $1940,383^{\mathrm{AL}}$, p. 1235-1245. In P. H. A. Sneath, N. S. Mair, M. E. Sharpe, and J. G. Holt (ed.), Bergey's manual of systematic bacteriology, vol 2. Williams \& Wilkins, Baltimore.

14. Skalka, B., J. Smola, and K. Elischerova. 1982. Routine test for in vitro differentiation of pathogenic and apathogenic Listeria monocytogenes strains. J. Clin. Microbiol. 15:503-507.

15. Smola, J., V. Katerov, and C. Schalén. 1994. Haemolytic and phospholipase C (PLC) activities of Rhodococcus equi. J. Appl. Bacteriol. 77:325-333.

16. Suárez, G., and L. Domínguez. 1995. Utility of the haemolytic phenotype in the recognition and identification of Listeria monocytogenes. The CAMP test, p. 9-14. In G. J. Arnold, P. S. Sutherland, and E. A. Szabo (ed.), Detection, identification and typing of $L$. monocytogenes in foods. Listeria methods workshop manual. Perth, Australia.

17. Vázquez-Boland, J. A., L. Domínguez, J. F. Fernández, E. F. RodríguezFerri, V. Briones, M. Blanco, and G. Suárez. 1990. Revision of the validity of CAMP test for Listeria identification. Proposal of an alternative method for the determination of haemolytic activity by Listeria strains. Acta Microbiol. Hung. 37:201-206

18. Vázquez-Boland, J. A., L. Domínguez, J. F. Fernández-Garayzábal, and G. Suárez. 1992. Listeria monocytogenes CAMP reaction. Clin. Microbiol. Rev. 5:343.

19. Vela, A. A. 1996. Ph.D. thesis. University of Zaragoza, Zaragoza, Spain.

20. Weaver, R. E. 1989. Morphological, physiological, and biochemical characterization, p. 39-43. In G. L. Jones (ed.), Isolation and identification of Listeria monocytogenes. Centers for Disease Control, Atlanta. 